\title{
RAIL SURFACE MEASUREMENT AND MULTI-SCALE MODELING OF WHEEL-RAIL CONTACT
}

\author{
Mohammad Mehdi Alemi \\ Virginia Tech \\ Department of Mechanical Engineering \\ Center for Tire Research (CenTiRe) \\ Blacksburg, VA, United States 24061 \\ Email: malemi@vt.edu
}

\author{
Saied Taheri \\ Virginia Tech \\ Department of Mechanical Engineering \\ Center for Tire Research(CenTiRe) \\ Blacksburg, VA, United States 24061 \\ Email: staheri@vt.edu
}

\author{
Mehdi Ahmadian \\ Virginia Tech \\ Department of Mechanical \\ Engineering \\ Rail Technologies Laboratory \\ (RTL) \\ Blacksburg, VA, United States \\ 24061 \\ Email: ahmadian@vt.edu
}

\section{ABSTRACT}

In railroad industries, one of the most important concepts is the ability to model and estimate the friction between the rail and the wheels. Overall, creating a general friction model is a challenging task because friction is influenced by different factors such as surface metrology, properties of materials in contact, surface contamination, flash temperature, normal load, sliding velocity, surface deformation, inter-surface adhesion, etc. Moreover, increase in the number of interfering factors in the process would add to the complexity of the friction model. Therefore, reliable prediction of the friction both theoretically and empirically is sensitive to how the model parameters are measured. Due to both safety and energy concerns, any attempts towards a better understanding of wheel/rail contact are considered important for the railroad industry. In this study, surface characteristics of four rail surfaces were measured at 20 microns over a rectangular area using a portable Nanovea Jr25 optical surface profilometer and the results were studied using various statistical procedures and Fractal theory. Furthermore, a 2D rectangular area was measured in this study because 1D height profile doesn't capture all the necessary statistical properties of the surface. For surface roughness characterization, the $3 \mathrm{D}$ parameters such as root-mean-square (RMS) height, skewness, kurtosis and other important parameters were obtained according to ISO 25178 standard. To verify the statistical results and fractal analysis, a British Pendulum Skid Resistance Tester was used to measure the average sliding coefficients of friction based on several experiments over a $5 \mathrm{~cm}$ contact length for all four rail sections. The results supported this fact that the rail surface with lower fractal dimension number has the lower friction. In effect, the larger fractal dimension number simply would add more microtexture features to the contact surface which potentially increases the friction. This paper will discuss the results and the next steps towards a better understanding of the friction potential between the wheels and the track.

\section{INTRODUCTION}

All efforts taken towards developing a universal model capable of estimating friction has been led to frustration due to multiplicity of effective variables and different initial and 
boundary conditions of the systems. In railroad industries, a good friction model may increase the life time of parts and reduce the overall energy consumption [1]. Therefore, the intelligent friction control is becoming a worldwide demand from both industrial consumers and producer [1]. Historically, the most important barrier in friction modeling was the lack of a perfect contact mechanics theory. The first contact mechanics theory was published by Hertz in 1881 [2]. He introduced the frictionless contact between elastic solids with smooth surface profiles. He found out that the contact surfaces could be modeled as parabolic and he developed his model assuming that the randomly rough but nominally flat surfaces could be modeled as a series of spherical bumps with equal height and radius of curvature. Due to Hertz's idealized model for randomly rough surfaces, Archard described his model in 1957 [3]. He claimed that his model reconsiders the physics of contact appropriately and the randomly rough surfaces could be illustrated including the small spherical bumps on the top of larger spherical bumps. Nine years later in 1966, Greenwood and Williamson introduced their model where the rough surfaces could be modeled as spherical bumps, which have equal radius of curvature but the distribution of heights is a Gaussian distribution [4]. Bush, Gibson and Thomas proposed a new model in 1975 emphasizing that the surface summits can be estimated as paraboloids and then the Hertz contact theory can be applied to them [5]. All of the above contact mechanics theories have two restrictions. First, the real area of contact should be much smaller than the nominal area of contact. This means that these contact models are valid for low squeezing force. Secondly, they didn't consider the influence and propagation of deformation fields for asperities. However, the results of all aforementioned contact models, except for the Hertz model, indicate that the real area of contact is proportional to normal load. In 2001, Persson introduced his contact mechanics theory where his model was the most comprehensive model compared to the previous ones [6,7]. Persson also considered the elastic coupling of asperities and his model was valid for all normal loads [8]. In persson's model, the surface roughness power spectrum plays the key role and it can be calculated using the surface height profile.

The surface texture is an important concern in calculating the surface friction and wear because surface roughness can directly change the lateral motion of surface when it is in contact with other surface. In recent years, the roughness parameters and topography are used to predict the frictional behavior of the surfaces. The standard surface roughness parameters including average height profile, root mean square of profile and maximum height profile are not enough in describing the frictional behavior of the surfaces [1]. This is because some very different surfaces shared similar standard roughness parameters [1]. As a result, other parameters including skewness and kurtosis will become more important especially because a lot of new models consider the height distributions as Gaussian [1,9]. If a distribution is Gaussian, the kurtosis and skewness are three and zero, respectively. For example, see Tayebi et al. (2003) Figure 1, page 492 [9].

Based on contact mechanics theories, the real area of contact is proportional to squeezing force. Therefore, the surfaces with positive skewness and kurtosis higher than 3 , experience lower friction and are good candidates for reduced friction applications [9].

In this paper, the roughness parameters have been introduced using ISO 25178 standard. ISO 25178 standard proposes more surface roughness parameters and hence, it illustrates a better description for the surface. In addition to standard surface roughness parameters, skewness and kurtosis are becoming important in ISO 25178. In general, zero skewness shows that the surface height distribution is symmetrical. A surface with negative skewness means that it has a lower number of peaks or it has deep scratches [1]. However, a surface with positive skewness is peakier or it has more filled valleys [1]. Kurtosis shows how the profile density sharpness has been distributed. If the kurtosis value is higher than 3 , the surface has high peaks and low valleys, however, if the surface has a kurtosis value lower than 3 , the surface has low peaks and low valleys.

In considering the surface texture parameters of rail sections, it is impossible to run the surface profilometry over a very large sectional area of rail. This drawback associates with the limitation of surface profilometry devices. In addition, it prolongs the surface profile measurements and post-processing calculations. Hence, a sample section of rail profile is measured and the surface roughness parameters are calculated. According to the surface fractal theory, results from small sections of profile can be generalized to the larger profiles for certain range scales. In 1967, Mandelbrot reported that many surfaces are approximately self-affine fractal [10]. In his book "the fractal geometry of nature", he mentioned that a surface is called fractal if a section of surface magnifies, it looks similar to the original whole surface $[8,11]$. This crucial invention is very important because for the fractal surfaces, the surface statistical properties remain the same in case of changing the scale. This is also valid for the investigated rail sections in this research for certain range scales. Based on the fractal analysis for rail sections, a parameter called fractal dimension number is introduced as below and this value is used to compare the rail sections:

$D_{f}=-\frac{\log N}{\log \varepsilon}$

Where $D_{f}$ is fractal dimension number, $\mathrm{N}$ is the number of sticks and $\varepsilon$ is the scaling factor [11].

Finally, In this research, a British Pendulum Skid Resistance Tester was used to find the coefficients of friction for the rail sections and compare them with dimension fractal numbers and statistical surface roughness parameters. 


\section{EXPERIMENTAL PROCEDURES}

In this study, the surface profiles, were, measured for four different rail sections provided by Norfolk Southern, The rail sections were labeled with capital alphabets A through D. The rail sections are shown in Fig. 1.

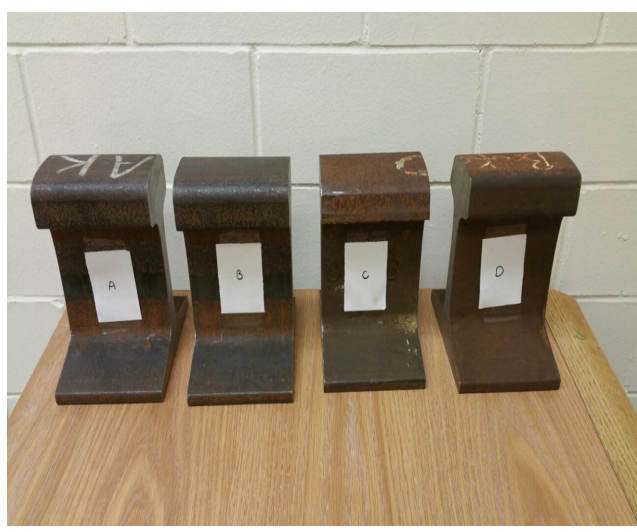

Figure 1: Measured rail sections

To do the surface profilometry, a portable Nanovea JR25 optical surface profilometer was used and the surface characteristic of rail sections at 15 microns over a rectangular area were calculated. The Jr25 optical surface profilometer uses an optical measurement principle which is called axial chromatism. This technique utilizes a white light source in which light can pass through an objective lens with a high degree of chromatic aberration. The objective lens's refractive index varies in relation to the wavelength of the light. In operation, each separate wavelength of the incident white light refocus at a different distance from the lens (different height). When the measured sample is within the range of possible heights, a single monochromatic point is focused to form the image. Due to the confocal configuration of the system, only the wavelength in focus is able to pass through the spatial filter with high frequency which causes all other wavelength to be out of focus. Pictures of JR25 optical surface profilometer and a wiring setup of JR25 optical profilometer for surface height measurement are shown in Fig. 2 and Fig. 3.

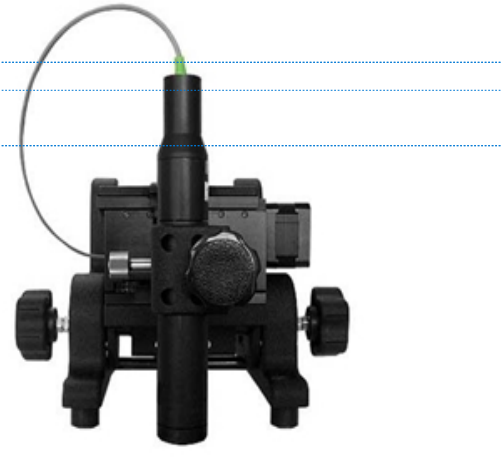

Figure 2: JR25 optical surface profilometer

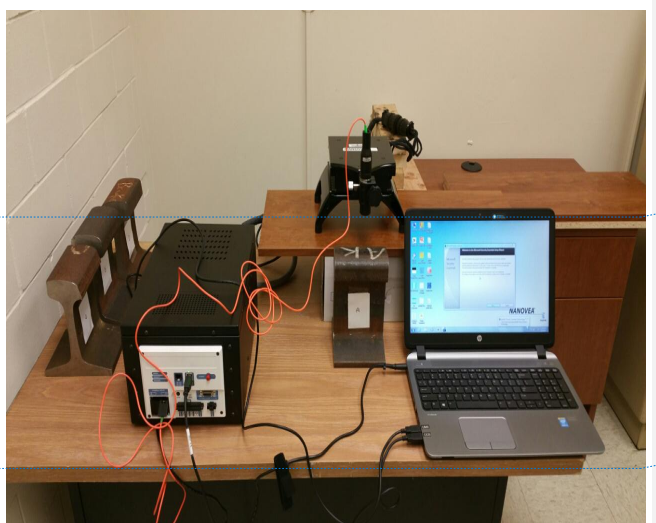

Figure 3: Wiring setup of Jr25 profilometer for height measurement

In order to compare the statistical results of surface profilometry and fractal dimension numbers, a series of experiments were performed to determine the real coefficient of friction for all rail sections. For this part, a British Pendulum Skid Resistance Tester was used to measure the coefficient of friction. Initially the British Pendulum was carefully calibrated and set to zero for each experiment. Then, to better estimate the coefficient of friction for the rail sections, seven experiments were run for each rail section and the average coefficient of friction was calculated. A picture of a British Pendulum is shown in Fig. 4. The British pendulum works based on the friction resistance between the rubber and the desired material surface. The pendulum is placed on the top of the rail surface

Deleted: ometry
Deleted: as
Deleted: Railway
Deleted: picture of all
Deled: is

Deleted: Picture of four

Deleted:

Deleted:

Deleted: as

Deleted: four

Deleted: announced for each rail 
from which coefficient of friction was targeted. The contact length of the surface can be adjusted and levelled manually. Next, the pendulum was released to swing down until the rubber slider contacted the surface of the rail. The position of the pointer on the British pendulum has been scaled from 0 to 150. The numbers on the pointer readings shows the resistance to skidding of the test surface.

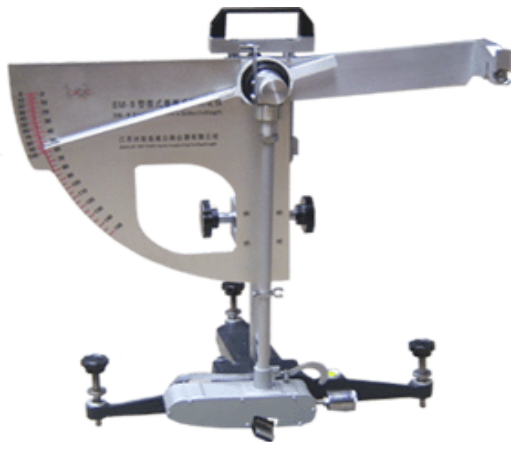

Figure 4: Picture of British Pendulum Skid Resistance Tester

\section{RESULTS AND DISCUSSIONS}

After finishing surface profilometry with JR25 optical profilometer, the $3 \mathrm{D}$ views of surface profiles are plotted. These $3 \mathrm{D}$ graphs display the topography within an interactive, realtime $3 \mathrm{D}$ visualization. In Fig. 5 the $3 \mathrm{D}$ height profiles have been plotted while the angle has been set to $45^{\circ}$ right view.

As discussed earlier, for surface roughness characterization, ISO 25178 height parameters were calculated. ISO 25178 is the first international standard taking into account the specification and measurement of 3D surface texture. In particular, the standard defines 3D surface texture parameters and the associated specification operators. The height profile parameters in ISO 25178 involve only the statistical distribution of height values along the $\mathrm{z}$ axis including root mean square height of the surface (Sq), skewness of height distribution (Ssk), kurtosis of height distribution (Sku), maximum height of peaks (Sp), maximum height of valleys or pit (Sv), maximum height of the surface $(\mathrm{Sz})$ and arithmetical mean height of the surface (Sa). The results of all ISO 25178 parameters including their unites for the rail sections have been shown in Table 1.

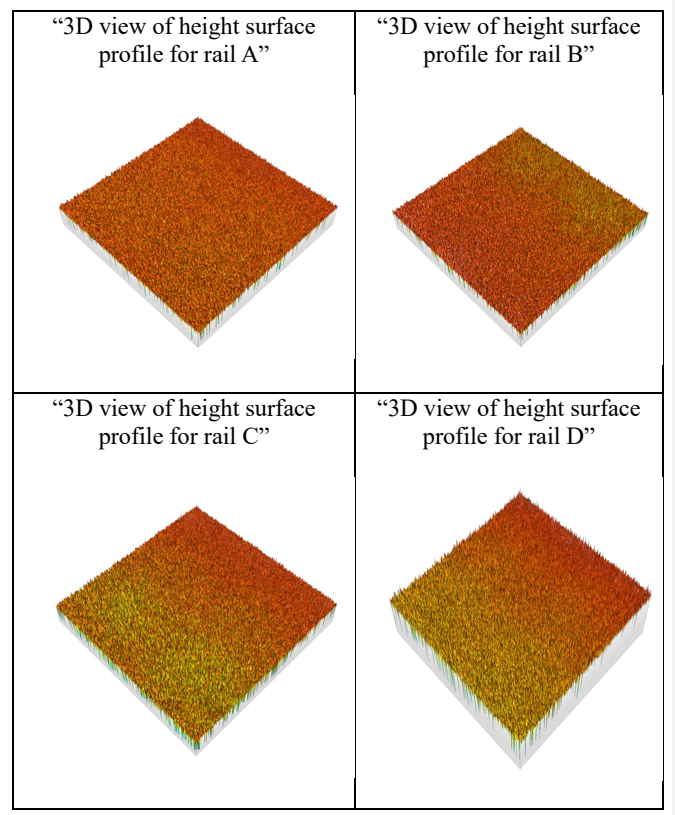

Figure 5: 3D view of height surface profile for rail sections

Table 1: Height parameters for all rail sections based on ISO 25178 standard

\begin{tabular}{|c|c|c|c|c|}
\hline \multicolumn{5}{|c|}{ Height Parameters - ISO 25178 Standard } \\
\hline $\begin{array}{c}\text { Statistical } \\
\text { Parameters }\end{array}$ & Rail A & Rail B & Rail C & Rail D \\
\hline$S q$ & $\begin{array}{c}16.801 \\
(\mu \mathrm{m})\end{array}$ & $\begin{array}{c}26.153 \\
(\mu \mathrm{m})\end{array}$ & $\begin{array}{c}27.700 \\
(\mu \mathrm{m})\end{array}$ & $\begin{array}{c}16.478 \\
(\mu \mathrm{m})\end{array}$ \\
\hline$S s k$ & -2.478 & -1.141 & -0.888 & -2.311 \\
\hline$S k u$ & 9.549 & 2.775 & 2.166 & 10.024 \\
\hline$S p$ & 39.584 & 46.133 & 51.825 & 46.532 \\
$(\mu \mathrm{m})$ & $(\mu \mathrm{m})$ & $(\mu \mathrm{m})$ & $(\mu \mathrm{m})$ \\
\hline$S v$ & 86.366 & 79.384 & 73.394 & 89.761 \\
& $(\mu \mathrm{m})$ & $(\mu \mathrm{m})$ & $(\mu \mathrm{m})$ & $(\mu \mathrm{m})$ \\
\hline$S z$ & 125.950 & 125.517 & 125.220 & 136.293 \\
& $(\mu \mathrm{m})$ & $(\mu \mathrm{m})$ & $(\mu \mathrm{m})$ & $(\mu \mathrm{m})$ \\
\hline$S a$ & 10.119 & 20.928 & 23.647 & 10.382 \\
$(\mu \mathrm{m})$ & $(\mu \mathrm{m})$ & $(\mu \mathrm{m})$ & $(\mu \mathrm{m})$ \\
\hline & \multicolumn{4}{|c}{}
\end{tabular}

As it can be seen in table 1, Rail A and Rail D have very similar root mean square $\left(S q_{A} \simeq S q_{\mathrm{D}}\right)$ and arithmetic mean height $\left(S a_{A} \simeq S a_{\mathrm{D}}\right.$ ). Therefore, to compare the friction of these two rails, the values of skewness and kurtosis could be
Deleted: , 
compared. Both Rail A and Rail D have negative skewness of 2.478 and -2.311 , respectively. The skewness of Rail D is higher (more positive) compared to Rail A. The corresponding kurtosis for Rail A and Rail D are 9.549 and 10.024 and so Rail D has higher kurtosis value compared to Rail A. Since rail D has higher kurtosis and more positive skewness compared to rail $\mathrm{A}$, then, rail $\mathrm{D}$ has lower friction compared to Rail $\mathrm{A}$ $\left(\mu_{D}<\mu_{A}\right)$ and apparently it is older than rail $\mathrm{A}$ as well.

Rail B and Rail $\mathrm{C}$ have also close root mean square $\left(S q_{\mathrm{B}} \simeq S q_{\mathrm{C}}\right)$ and arithmetic mean height $\left(S a_{\mathrm{B}} \sim S a_{\mathrm{C}}\right)$.

Based on the results shown in Table 1, rail $\mathrm{B}$ and rail $\mathrm{C}$ have kurtosis values of 2.775 and 2.166 , respectively. These kurtosis values are lower than 3 . Therefore, the values of skewness can be used to compare the friction for rail B and rail C. Rail B and rail $\mathrm{C}$ have negative skewness of -1.141 and -0.888 , respectively and so rail $\mathrm{C}$ has more positive skewness value compared to rail B. Consequently, the statistics in table 1 result in lower friction for Rail C $\left(\mu_{C}<\mu_{B}\right)$.

For the last comparison, it is mentioned that both rail A and rail $\mathrm{C}$ have negative skewness. Therefore, to compare the friction for these two rail sections, the root mean square, arithmetic mean height and kurtosis values are preferably used. Based on statistics in Table 1, it is noticed that the root mean square and arithmetic mean height for rail $\mathrm{C}$ are higher than rail $\mathrm{A}$ $\left(S q_{\mathrm{C}}>S q_{\mathrm{A}}, S a_{\mathrm{C}}>S a_{\mathrm{A}}\right)$. Also the kurtosis value for rail A is almost four times larger than kurtosis value for rail $\mathrm{C}$ $\left(S k u_{\mathrm{A}}=9.549>>S k u_{\mathrm{C}}=2.166\right)$. So, it can be concluded that statistical results show lower friction for rail A compared to rail $\mathrm{C}\left(\mu_{A}<\mu_{C}\right)$. All of these conclusions show that rail $\mathrm{B}$ has the highest friction(the newest rail section) and rail $\mathrm{D}$ has the lowest friction(the oldest rail section).

In the second part of this study, fractal analysis was done for all rail sections and the fractal dimension numbers were reported for each rail sections. The fractal dimension $\left(D_{f}\right)$ is an index used to characterize the fractal patterns by quantifying their complexity as a ratio of the change in detail to the change in scale [11]. In other words, the fractal dimension describes the complexity of the surface in the form of a single number. The fractal dimension has also been characterized as a measure of the space-filling capacity of a pattern that tells how a fractal scales differently from the space it is embedded in [11]. Despite of Euclidean dimension which is always an integer number, a fractal dimension does not have to be an integer. The ability to calculate and estimate the fractal dimension will help us for better understanding and predicting the behavior of the patterns or sets. The fractal dimension values for different rail sections have been calculated using encloses boxes method. In the last step of the experiments, the coefficients of friction have been experimentally obtained using the British Pendulum shown in Fig. 4. A summary of important statistical surface roughness parameters aligned with fractal dimension number and experimental coefficient of friction for each rail section is introduced in Table 2.

Table 2: Comparison of rail section based on important statistical parameters, fractal dimension number and experimental coefficient of friction

\begin{tabular}{|c|c|c|c|c|c|c|}
\hline $\begin{array}{c}\text { Rail } \\
\text { Sections }\end{array}$ & $S q$ & $S a$ & $S s k$ & $S k u$ & $D_{f}$ & $\mu$ \\
\hline Rail A & 16.801 & 10.119 & -2.478 & 9.549 & 2.966 & 0.31 \\
\hline Rail B & 26.153 & 20.928 & -1.141 & 2.775 & 2.981 & 0.34 \\
\hline Rail C & 27.700 & 23.647 & -0.888 & 2.166 & 2.980 & 0.33 \\
\hline Rail D & 16.478 & 10.382 & -2.311 & 10.024 & 2.938 & 0.17 \\
\hline
\end{tabular}

Based on fractal dimension numbers in Table 2, it can be concluded that the rail surface with lower fractal dimension number has the lower friction as well. In effect, the larger fractal dimension number simply would add more microtexture features to the contact surface which potentially increases the friction. As it can be seen in Table 2, rail B and rail D have the highest (2.981) and lowest dimension number (2.938) and consequently highest and lowest friction. Also, experimental results for coefficient of friction using the British Pendulum shows that the fractal analysis, statistical surface roughness parameters and experimental results are in agreement with each other.

\section{CONCLUSIONS}

In this study, initially the statistical surface roughness parameters were obtained for four different rail sections provided by Norfolk Southern. The surface characteristic analysis was started with surface profile measurement using a portable JR25 Nanovea optical surface profilometer. Then, the 3D results were translated to various statistical parameters based on ISO 25178 standards including arithmetic mean height $(S a)$, root mean square $(S q)$, skewness $(S s k)$, kurtosis $(S k u)$ and etc. Next, the surface characteristic was related to surface topography for further friction investigation. The two critical parameters in comparing the surfaces were kurtosis and skewness and it was found that the surfaces with higher kurtosis values and more negative skewness will lead to low friction cases, It is worth mentioning that for all of the investigated, rail sections, the skewness values had negative values which means that the surfaces have been designed for reduced friction applications. Also for the cases that the kurtosis and skewness have very similar values, the important parameter for investigation is the root mean square. The statistical results showed that rail $\mathrm{B}$ had the highest friction and rail $\mathrm{D}$ had the lowest friction. Rail $\mathrm{C}$ had also higher friction compared to rail A. Moreover, the fractal analysis was performed based on surface profilometry for all rail sections. The result of fractal analysis was summarized to fractal dimension numbers which supported the statistical results. In the last step, to make sure that the theoretical results are in agreement with real experimental results, a British pendulum

\begin{tabular}{l} 
Deleted: Railway \\
Deleted: ometry \\
Deleted: the \\
Deleted: has been \\
Deled: [1] \\
\hline
\end{tabular}


skid resistance tester was used to measure the friction of coefficient over a contact of length of $5 \mathrm{~cm}$. The results of British pendulum test was consistent with the statistical and fractal results. In sum, the surfaces with higher fractal dimension numbers experience higher friction because of the more microtexture features added to the contact surface.

\section{NOMENCLATURE}

In order to better understanding of statistical surface roughness parameters in ISO 25178 standard and also other important parameters in the paper, the following nomenclature table 3 has been provided.

Table 3: Nomenclature of different parameters

\begin{tabular}{|c|c|}
\hline$S q$ & Root mean square height of surface \\
\hline$S s k$ & Skewness of surface height distribution \\
\hline$S k u$ & Kurtosis of surface height distribution \\
\hline$S p$ & Maximum height of peaks \\
\hline$S v$ & Maximum height of valleys \\
\hline$S z$ & Maximum height of surface \\
\hline$S a$ & Arithmetic mean height of surface \\
\hline$D_{f}$ & Fractal dimension number \\
\hline$\mu$ & Coefficient of friction \\
\hline$\varepsilon$ & Scaling factor \\
\hline
\end{tabular}

\section{ACKNOWLEDGMENTS}

The authors would like to acknowledge the contribution of Norfolk Southern Railway by providing the four rail sections.

\section{REFERENCES}

1. Sedlaček, M., Vilhena, L. M. S., Podgornik, B., \& Vižintin, J. (2011). Surface topography modelling for reduced friction. Strojniški vestnik-Journal of Mechanical Engineering, 57(9), 674-680.

2. Hertz, Heinrich. "On the contact of elastic solids." $J$. reine angew. Math 92.156-171 (1881): 110.

3. Archard, J. F. (1957, December). Elastic deformation and the laws of friction. In Proceedings of the Royal Society of London A: Mathematical, Physical and Engineering Sciences (Vol. 243, No. 1233, pp. 190205). The Royal Society.

4. Greenwood, J. A., \& Williamson, J. B. P. (1966, December). Contact of nominally flat surfaces. In Proceedings of the Royal Society of London A Mathematical, Physical and Engineering Sciences (Vol. 295, No. 1442, pp. 300-319). The Royal Society.
5. Bush, A. W., Gibson, R. D., \& Thomas, T. R. (1975). The elastic contact of a rough surface. Wear, 35(1), $87-111$.

6. Persson, B. N. (2001). Theory of rubber friction and contact mechanics. The Journal of Chemical Physics, 115(8), 3840-3861.

7. Persson, B. N. J., Bucher, F., \& Chiaia, B. (2002). Elastic contact between randomly rough surfaces: comparison of theory with numerical results. Physical Review B, 65(18), 184106.

8. Persson, B. N. (2006). Contact mechanics for randomly rough surfaces.Surface Science Reports, 61(4), 201-227.

9. Tayebi, N., \& Polycarpou, A. A. (2004). Modeling the effect of skewness and kurtosis on the static friction coefficient of rough surfaces. Tribology international, 37(6), 491-505.

10. Mandelbrot, B. B. (1967). How long is the coast of Britain. Science, 156(3775), 636-638

11. Mandelbrot, B. B. (1983). The fractal geometry of nature (Vol. 173). Macmilla 
